\title{
CONTRIBUIÇÃO AO CONHECIMENTO DA ICTIOFAUNA DO MANGUEZAL DE CACHA PREGOS, ILHA DE ITAPARICA, BAIIA DE TODOS OS SANTOS, BAHIA
}

\author{
Paulo Roberto Duarte Lopes ${ }^{1}$ \\ Jailza Tavares de Oliveira-Silva ${ }^{1}$ \\ Andréa Silene Alves Ferreira-Melo ${ }^{1}$
}

\begin{abstract}
CONTRIBUtion to KNOWLEDgE OF ICHTHYofauna OF MANGROVE CACHA Pregos, Itaparica island, Todos os Santos Bay, Bahia. A check-list of fishes collected in a mangrove in Cacha Pregos, south of Itaparic Island, State of Bahia, Brazil (about $13^{\circ} 07^{\prime} \mathrm{S}, 38^{\circ} 48^{\prime} \mathrm{W}$ ) from 1988-1989 and 1991-1992 is presented. Fifteen orders, 46 families and 85 species (Teleostei except one Chondrichthyes) were identified, represented specimens whose adults live in different marine ecossystems. KEY WORDS. Check-list, ichthyofauna, mangrove, Itaparica Island, Todos os Santos Bay, Bahia
\end{abstract}

O estado da Bahia possui um litoral de $1.188 \mathrm{~km}$ representando $13,2 \%$ da costa brasileira e que apresenta grande número de estuários cujas bordas caracterizam-se pela formação de mangues arbóreos, ricos em numerosas espécies utilizáveis na alimentação e, portanto, de relevância sócio-econômica; dentre estas regiões estuarinas destacam-se principalmente a Baía de Todos os Santos e Baía de Camamu (BAHIA PESCA 1994).

A Baía de Todos os Santos ocupa cerca de $800 \mathrm{~km}^{2}$ e concentra atividades portuárias e industriais que afetam, sobretudo, a ecologia da região em decorrência da poluição de origem urbana e industrial, acentuada por um intenso processo de ocupação desordenada que vem destruindo os manguezais (SANTOS 1973; BAHIA PESCA 1994).

\section{MATERIAL E MÉTODOS}

A Ilha de Itaparica é a maior da Baía de Todos os Santos e situa-se em sua entrada e a localidade de Cacha Pregos encontra-se no seu extremo sul (cerca de $13^{\circ} 07^{\prime} \mathrm{S}, 38^{\circ} 48^{\prime} \mathrm{W}$, figura 1 ), apresentando extensa área de manguezal em excelente estado de conservação onde a população local realiza uma pesca de subsistência principalmente de crustáceos e moluscos (LOPES \& MIRANDA 1995).

1) Laboratório de Ictiologia, Departamento de Ciências Biológicas, Universidade Estadual de Feira de Santana. Campus Universitário BR 116, Km 3, 44031-460 Feira de Santana, Bahia, Brasil. 


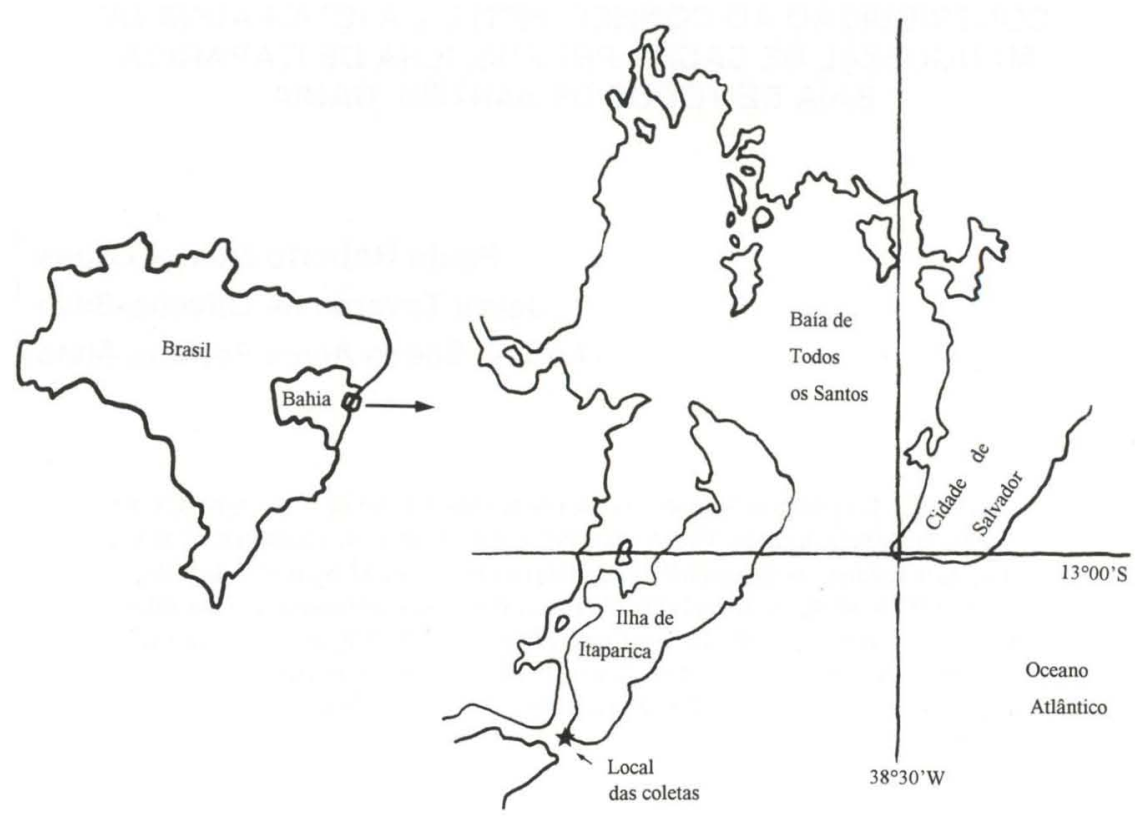

Fig. 1. Mapa da região indicando o local da coleta.

As coletas foram realizadas mensalmente em uma área do manguezal na localidade de Cacha Pregos durante a baixa-mar com o auxílio de uma pequena rede de arrasto manual, no período de outubro de 1988 a dezembro de 1989 (exceto nos meses de janeiro, fevereiro e março de 1989) e no período de agosto de 1991 a dezembro de 1992 (exceto nos meses de fevereiro, março, julho e outubro de 1992).

Ainda no campo, após serem coletados, os exemplares foram fixados em formol a $10 \%$. Material representativo de cada espécie capturada encontra-se depositado na coleção do Laboratório de Ictiologia (LIUEFS) - Departamento de Ciências Biológicas, Universidade Estadual de Feira de Santana (UEFS, Bahia), conservado em álcool a $70 \%$.

Para determinação do comprimento padrão utilizou-se um paquímetro com precisão de 0,05 mm com base no critério de FIGUEIREDO \& MENEZES (1978). Para o material relativo às famílias Torpedinidae, Muraenidae, Ophichthidae e Congridae optou-se pela determinação do comprimento total conforme definido também por FIGUEIREDO \& MENEZES (1978).

A seqüência e a grafia dos nomes das ordens e famílias seguem NELSON (1994). Os nomes e das espécies seguem dispostos em ordem alfabética. A bibliografia consultada para a identificação à nível genérico e/ou específico é apresentada ao lado do nome da respectiva família. 


\section{RESULTADOS}

Para cada espécie identificada são apresentados o número de registro do lote na coleção do LIUFS e seu respectivo número de exemplares bem como seu comprimento (total ou padrão) ou seu intervalo.

Entre o material coligido foram identificadas 15 ordens, 46 famílias, 71 gêneros e 85 espécies. Apenas uma espécie de Chondrichthyes foi coletada sendo as demais representadas por Teleostei.

Devido às diferenças observadas entre as diagnoses apresentadas, para algumas espécies, na literatura consultada e as características de diversos exemplares examinados, optou-se por manter suas identificações somente à nível genérico.

\section{Chondrichthyes}

Rajiformes

Torpedinidae (FIGUEIREDO 1977)

Narcine brasiliensis (Olfers, 1831) - LIUFS 462 (1: 91,0 mm)

\section{Actinopterygii}

Anguilliformes

Muraenidae (FIGUEIREDO \& MENEZES 1978)

Gymnothorax ocellatus Agassiz, 1831 - LIUFS 202 (1: 276,0 mm), LIUFS $220(1: 400,0 \mathrm{~mm})$

Ophichthidae (FIGUEIREDO \& MENEZES 1978)

Ophichthus ophis (Linnaeus, 1758) - LIUFS 201 (1: 368,0 mm)

Congridae (FIGUEIREDO \& MENEZES 1978)

Ariosoma sp. - LIUFS 203 (5: 201,0-251,0 mm), LIUFS 269 (1: 201,0 mm) Clupeiformes

Engraulidae (FIGUEIREDO \& MENEZES 1978; WHITEHEAD et al. 1988)

Anchoa sp. - LIUFS 045 (19: 50,4-71,4 mm), LIUFS 089 (2:26,3-29,5 mm),

LIUFS 185 (4: 33,3-42,5 mm), LIUFS 193 (5: 44,4-66,3 mm), LIUFS 196 (6: 32,5-54,2 mm), LIUFS 241 (5: 56,4-61,3 mm), LIUFS 246 (1: 45,1 mm), LIUFS $250(2: 43,5-70,2 \mathrm{~mm})$, LIUFS $255(3: 57,6-61,1 \mathrm{~mm})$, LIUFS 271 (4: 57,9-59,7 mm), LIUFS 280 (4: 29,8-32,6 mm), LIUFS 302 (1: 51,1 mm), LIUFS 303 (2: 45,6-53,9 mm), LIUFS 313 (2: 57,1-60,9 mm), LIUFS 455 (1: 75,3 mm), LIUFS $466(1: 56,7 \mathrm{~mm})$

Anchoviella lepidentostole (Fowler, 1911) - LIUFS 120 (4: 57,4-62,6 mm) Lycengraulis grossidens (Agassiz, 1829) - LIUFS 104 (1: 141,2 mm), LIUFS 205 (1: 120,9 mm)

Clupeidae (Figueiredo \& MENEZes 1978; Whitenead 1985)

Harengula jaguana Poey, 1865 - LIUFS 050 (8: 49,2-94,4 mm), LIUFS 085 (2: 97,6-103,4 mm), LIUFS 194 (6: 40,6-56,4 mm), LIUFS 299 (1: 42,1 $\mathrm{mm})$

Lile piquitinga (Schreiner \& Ribeiro, 1903) - LIUFS 166 (1: 69,6 mm), LIUFS 182 (3: 62,1-74,9 mm), LIUFS 301 (1: 70,5 mm) 
Opisthonema oglinum (Lesueur, 1818) - LIUFS 046 (6: 49,2-71,6 mm) Aulopiformes

Synodontidae (FIGUEIREDO \& MENEZES 1978)

Synodus foetens (Linnaeus, 1766) - LIUFS 080 (1: 107,6 mm), LIUFS 175 (1: 194,0 mm), LIUFS 200 (1: 154,2 mm)

Trachinocephalus myops (Forster, 1801) - LIUFS 039 (1: 126,1 mm) Batrachoidiformes

Batrachoididae (FIGUEIREDO \& MENEZES 1978; FISCHER 1978)

Thalassophryne sp. - LIUFS 242 (2: 43,8-68,8 mm), LIUFS 305 (1: 112,6 $\mathrm{mm})$, LIUFS $478(1: 109,3 \mathrm{~mm})$

Lophiiformes

Antennariidae (FigueIREdo \& MENEZES 1978; PIETSCH 1984)

Antennarius striatus (Shaw \& Noder, 1794) - LIUFS 098 (1: 68,6 mm)

Ogcocephalidae (FIGUEIREDO \& MENEZES 1978)

Ogcocephalus vespertilio (Linnaeus, 1758) - LIUFS 070 (1: 61,4 mm), LIUFS 086 (1: 116,1 mm), LIUFS 099 (3: 67,4-82,7 mm), LIUFS 209 (1: 82,1 mm), LIUFS 233 (2: 106,9-145,9 mm), LIUFS 243 (1: 123,3 mm), LIUFS 253 (1: 101,1 mm), LIUFS 275 (1: 86,6 mm)

Mugiliformes

Mugilidae (Menezes \& Figueiredo 1985)

Mugil curema Valenciennes, 1836 - LIUFS 088 (9: 25,6-39,6 mm), LIUFS 219 (1: 95,8 mm), LIUFS 225 (1: 86,1 mm), LIUFS 277 (1: 144,5 mm), LIUFS 289 (1: 68,7 mm), LIUFS 481 (1: 68,0 mm)

Mugil trichodon Poey, 1876 - LIUFS 211 (1: 61,9 mm), LIUFS 489 (1: 84,4 $\mathrm{mm})$

Atheriniformes

Atherinidae (FIGUEIREDO \& MENEZES 1978)

Xenomelaniris brasiliensis (Quoy \& Gaimard, 1824) - LIUFS 168 (1: 66,9 $\mathrm{mm})$, LIUFS 190 (2: 80,4-81,5 mm)

Beloniformes

Belonidae (FIGUEIREDO \& MENEZES 1978)

Strongylura timucu (Walbaum, 1792) - LIUFS 108 (1: 294,0 mm), LIUFS $230(1: 289,0 \mathrm{~mm})$

Hermiramphidae (FIGUEIREDO \& MENEZES 1978)

Hyporhamphus unifasciatus (Ranzani, 1842) - LIUFS 051 (8: 149,3-183,0 $\mathrm{mm})$, LIUFS 257 (1: 99,5 mm)

Cyprinodontiformes

Poeciliidae (IHERING 1931)

Poecilia vivipara Bloch \& Schneider, 1801 - LIUFS 077 (1: $37,7 \mathrm{~mm})$, LIUFS 117 (1: 42,9 mm), LIUFS 235 (3: 46,5-51,2 mm), LIUFS 479 (3: 47,3-53,9 mm)

Gasterosteiformes

Syngnathidae (Figueiredo \& MENEZES 1980; BOHLKE \& CHAPLIN 1993)

Hippocampus reidi Ginsburg, 1933 - LIUFS 074 (1: 54,1 mm), LIUFS 268 
(1: 27,2 mm), LIUFS 282 (2: 27,7-37,4 mm), LIUFS 295 (1: 19,5 mm), LIUFS 312 ( $1: 44,6 \mathrm{~mm})$, LIUFS 454 ( $1: 24,6 \mathrm{~mm})$, LIUFS 474 (1: 27,4 $\mathrm{mm})$, LIUFS 499 (1: 65,1 mm), LIUFS $563(1: 76,9 \mathrm{~mm})$

Oostethus lineatus (Kaup, 1856) - LIUFS 490 (1: 78,7 mm)

Syngnathus pelagicus Linnaeus, 1758 - LIUFS 115 (1: 92,4 mm), LIUFS 116 (1: 74,4 mm), LIUFS 249 (2: 74,1-77,3 mm), LIUFS 287 (1: 76,6 $\mathrm{mm})$, LIUFS 292 (3: 67,4-77,8 mm), LIUFS 316 (1: 99,2 mm), LIUFS 451 (1: 73,3 mm), LIUFS $492(1: 100,1 \mathrm{~mm})$

Fistulariidae (FIGUEIREDO \& MENEZES 1980)

Fistularia tabacaria Linnaeus, 1758 - LIUFS 052 (1: 234,0 mm), LIUFS 165 (1: 103,8 mm), LIUFS 222 (1: 107,2 mm), LIUFS 252 (1: 145,0 mm), LIUFS 265 (1: 123,2 mm), LIUFS 272 (2: 91,4-115,6 mm), LIUFS $284(1: 119,6 \mathrm{~mm})$

Scorpaeniformes

Dactylopteridae (FIGUEIREDO \& MENEZES 1980)

Dactylopterus volitans (Linnaeus, 1758) - LIUFS 075 (1: 53,8 mm), LIUFS

112 (1: 47,6 mm), LIUFS 167 (1: 77,3 mm)

Scorpaenidae (FIGUEIREDO \& MENEZES 1980)

Scorpaena plumieri Bloch, 1789 - LIUFS 092 (1: 87,9 mm), LIUFS 260 (1:

$34,5 \mathrm{~mm})$, LIUFS 567 (2: 43,1-53,8 mm)

Triglidae (FIgUEIREDO \& MENEZES 1980)

Prionotus punctatus (Bloch, 1797) - LIUFS 040 (1: 99,9 mm), LIUFS 076

(1: 60,9 mm), LIUFS $186(1: 80,5 \mathrm{~mm})$, LIUFS $273(1: 107,4 \mathrm{~mm})$

Perciformes

Centropomidae (Figueiredo \& Menezes 1980; Rivas 1986)

Centropomus parallelus Poey, 1860 - LIUFS 094 (1: 175,0 mm), LIUFS

$113(1: 31,7 \mathrm{~mm})$, LIUFS $216(1: 113,5 \mathrm{~mm})$, LIUFS $296(1: 38,1 \mathrm{~mm})$,

LIUFS 315 (1: 53,9 mm), LIUFS 521 (2: 57,6-57,7 mm)

Centropomus undecimalis (Bloch, 1792) - LIUFS 281 (1: 61,6 mm)

Serranidae (Figueiredo \& Menezes 1980; Bohlke \& Chaplin 1993; HeEMSTRA \& RANDALL 1993)

Epinephelus itajara (Lichtenstein, 1822)-LIUFS 456 (1: 72,4 mm), LIUFS 463 (1: $81,8 \mathrm{~mm})$

Diplectrum radiale (Quoy \& Gaimard, 1824) - LIUFS 084 (1: 154,0 mm), LIUFS 199 (1: 147,0 mm), LIUFS 208 (1: 103,1 mm), LIUFS 215 (1: $69,4 \mathrm{~mm})$, LIUFS $566(1: 61,3 \mathrm{~mm})$

Mycteroperca bonaci (Poey, 1862) - LIUFS 114 (1: 71,3 mm), LIUFS 461

(2: 46,8-49,2 mm), LIUFS 519 (2: 34,2-40,5 mm)

Rypticus saponaceus (Bloch \& Schneider, 1801)-LIUFS 041 (1:61,6 mm),

LIUFS 053 (1: 101,4 mm), LIUFS 097 (1: 80,5 mm), LIUFS 227 (1:

$86,4 \mathrm{~mm})$, LIUFS 235 (1: 67,1 mm)

Serranus flaviventris (Cuvier, 1829) - LIUFS 107 (1:29,4 mm)

Carangidae (MENEZES \& Figueiredo 1980)

Caranx latus Agassiz, 1831 - LIUFS 078 (1: 41,1 mm), LIUFS 178 (2:

91,2-94,5 mm), LIUFS 188 (1: 85,4 mm), LIUFS 231 (1: 78,3 mm), 
LIUFS 248 (1: 39,9 mm), LIUFS 293 (1: 57,9 mm), LIUFS 310 (1: 44,6 $\mathrm{mm})$

Chloroscombrus chrysurus (Linnaeus, 1766) - LIUFS 054 (1: 108,1-132,1 mm), LIUFS 119 (2: 109,3-119,3 mm), LIUFS 192 (2: 30,5-34,4 mm) Oligoplites saurus (Bloch \& Schneider, 1801) - LIUFS 238 (1: 66,3 mm), LIUFS 297 (5: 21,6-30,6 mm), LIUFS 304 (2: 24,8-28,3 mm), LIUFS 319 (2: $36,1-54,7 \mathrm{~mm})$

Selene vomer (Linnaeus, 1758) - LIUFS 121 (1: 151,0 mm), LIUFS 171 (1: $31,6 \mathrm{~mm}$ ), LIUFS 210 (3: 34,8-45,4 mm), LIUFS 245 (1: 42,7 mm), LIUFS 480 (1: $37,3 \mathrm{~mm})$

Trachinotus carolinus (Linnaeus, 1766) - LIUFS 069 (2: 51,4-52,9 mm) Lutjanidae (MENEZES \& FiguEIREDO 1980; ALLEN 1985)

Lutjanus analis (Cuvier, 1828) - LIUFS 057 (1: 104,8 mm), LIUFS 102 (1: $66,4 \mathrm{~mm})$, LIUFS $169(1: 138,4 \mathrm{~mm})$

Lutjanus griseus (Linnaeus, 1758) - LIUFS 237 (1: 100,8 mm)

Lutjanus jocu (Bloch \& Schneider, 1801) - LIUFS 047 (3: 92,2-109,2 mm)

Lutjanus synagris (Linnaeus, 1758) - LIUFS 058 (6: 72,9-87,9 mm), LIUFS

073 (3: 43,8-47,8 mm), LIUFS 213 (1: 61,8 mm)

Ocyurus chrysurus (Bloch, 1791) - LIUFS 491 (1: 46,9 mm)

Gerreidae (MENEZES \& FigueIREDo 1980)

Diapterus rhombeus (Cuveir, 1829) - LIUFS 056 (4: 52,1-67,3 mm), LIUFS 229 (1: 60,4 mm), LIUFS 457 (1: 34,4 mm)

Eucinostomus argenteus (Baird \& Girard, 1854) - LIUFS 043 (1: 95,5 mm), LIUFS 181 (1: 77,5 mm), LIUFS 195 (3: 63,5-73,6 mm), LIUFS 217 (1: $111,1 \mathrm{~mm})$, LIUFS $300(2: 43,4-80,8 \mathrm{~mm})$

Eucinostomus gula (Cuvier, 1830) - LIUFS 042 (1: 74,2 mm), LIUFS 055 (1: 79,4 mm), LIUFS $079(1: 72,7 \mathrm{~mm})$, LIUFS $176(1: 73,8 \mathrm{~mm})$

Haemulidae (CERvigón et al. 1992; MENEZES \& FigUEIREDO 1980)

Haemulon sp. - LIUFS 072 (1: 80,3 mm)

Haemulon steindachneri (Jordan \& Gilbert, 1882) - LIUFS 218 (1: 95,6 $\mathrm{mm}$ )

Pomadasys croco (Cuvier, 1830) - LIUFS 317 (1: 40,9 mm), LIUFS 450 (1: $49,8 \mathrm{~mm})$

Polynemidae (MENEZES \& F $\mathrm{F}^{\text {IGUEIREDO }}$ 1985)

Polydactylus virginicus (Linnaeus, 1758) - LIUFS 118 (1: 85,6 mm)

Sciaenidae (MENEZES \& FigUEIREDO 1980)

Menticirrhus littoralis (Holbrook, 1860) - LIUFS 518 (1: 35,4 mm)

Stellifer stellifer (Bloch, 1790) - LIUFS 091 (4: 29,7-43,3 mm)

Mullidae (MENEZES \& FIGUEIREDO 1985)

Pseudupeneus maculatus (Bloch, 1793) - LIUFS 059 (19: 57,7-93,1 mm), LIUFS 264 (1: 53,3 mm)

Chaetodontidae (MENEZES \& FigUEIREDo 1985)

Chaetodon striatus Linnaeus, 1758 - LIUFS 305 (1: 32,4 mm), LIUFS 520 (2: $31,1-37,4 \mathrm{~mm})$, LIUFS 739 (1: 27,4 mm) 
Pomacanthidae (MeneZES \& Figueiredo 1985)

Pomacanths paru (Bloch, 1787) - LIUFS 095 (1: 81,1 mm)

Pomacentridae (MENEZES \& FigueiRedo 1985)

Abudefduf saxatilis (Linnaeus, 1758) - LIUFS 191 (3: 48,9-53,4 mm), LIUFS 308 (2: 29,6-30,7 mm)

Stegastes variabilis (Castelnau, 1855) - LIUFS 493 (3: 30,2-40,0 mm)

Scaridae (FISCHER 1978; MENEZES \& FIGUEIREDO 1985)

Sparisoma sp. - LIUFS 060 (5: 52,8-66,2 mm), LIUFS 183 (2: 80,2-89,4 mm), LIUFS 207 (1: 93,3 mm), LIUFS $476(1: 57,0 \mathrm{~mm})$

Gobiidae (MENEZES \& FIGUEIREDO 1985)

Bathygobius soporator (Valenciennes, 1837) - LIUFS 087 (4: 35,3-54,4 mm), LIUFS 111 (8: 50,2-76,3 mm), LIUFS 307 (1: 18,8 mm)

Gobionellus boleosoma (Jordan \& Gilbert, 1882) - LIUFS 110 (19: 13,7-27,2 mm), LIUFS 247 (3: 19,6-30,7 mm), LIUFS $267(1: 24,1 \mathrm{~mm})$ Gobionellus smaragdus (Valenciennes, 1837) - LIUFS 223 (1: 46,6 mm) Gobionellus stigmaticus (Poey, 1861) - LIUFS 334 (1: 37,9 mm)

Gobionellus stomatus Starks, 1913 - LIUFS 221 (7: 35,6-45,6 mm), LIUFS 251 (2: 54,4-59,6 mm), LIUFS 258 (1: $57,2 \mathrm{~mm})$, LIUFS 321 (7: 50,6-58,5 mm), LIUFS 501 (1: $57,8 \mathrm{~mm})$

Ephippidae (MENEZES \& FigUEIREDO 1985)

Chaetodipterus faber (Broussonet, 1782) - LIUFS 101 (1: 55,8 mm), LIUFS

177 (2: 48,1-58,9 mm)

Acanthuridae (FISCHER 1978)

Acanthurus chirurgus (Bloch, 1787) - LIUFS 488 (2: 27,9-28,6 mm)

Sphyraenidae (FISCHER 1978; MENEZES \& FigUEIREDO 1985)

Sphyraena barracuda (Walbaum, 1792) - LIUFS 240 (1: 87,9 mm), LIUFS 288 (4: 43,3-86,4 mm), LIUFS 309 (14: 35,9-110,6 mm)

Scombridae (COLLETTE \& NAUEN 1983)

Scomberomorus brasiliensis Collette, Russo \& Zavala-Camin, 1978 -LIUFS 170 (1: 147,2 mm)

Pleuronectiformes

Paralichthyidae (CARVAlHo 1968; Fischer 1978; SOARES 1978; TOPP \& HofF 1972)

Citharichthys cf. spilopterus Gunther, 1862 - LIUFS 049 (3: 72,2-89,5 mm), LIUFS 083 (16: 22,3-97,1 mm), LIUFS 100 (2: 64,7-92,6 mm), LIUFS 184 (3: 60,5-84,9 mm), LIUFS 274 (1: 126,6 mm), LIUFS 286 (1: 72,6 $\mathrm{mm})$, LIUFS 314 (1: $56,9 \mathrm{~mm}$ )

Paralichthys brasiliensis (Ranzani, 1840) - LIUFS 048 (1: 135,2 mm)

Syacium sp. - LIUFS 1616 (1: 55,4 mm)

Syacium cf. papillosum Linnaeus, 1758 - LIUFS 354 (1: 146,9 mm), LIUFS $1617(1: 121,8 \mathrm{~mm})$

Achiridae (CARVALHO 1968; FISCHER 1978; SOARES 1978)

Achirus sp. - LIUFS 096 (1: 37,9 mm), LIUFS 187 (1: 49,2 mm), LIUFS 224 (1: 85,9 mm), LIUFS 234 (1: 125,9 mm), LIUFS 261 (1: 42,5 mm), LIUFS 318 (1: $43,7 \mathrm{~mm})$ 
Cynoglossidae (CARVAlHo 1968; SOARES 1978)

Symphurus sp. - LIUFS 206 (1: 113,2 mm)

Tetraodontiformes

Balistidae (FISCHER 1978; CERVIGÓN et al. 1992)

Balistes vetula Linnaeus, 1758 - LIUFS 212 (1: 47,4 mm), LIUFS 236 (1: $46,9 \mathrm{~mm})$

Monacanthidae (FISHCER 1978; CERVIGÓN et al. 1992)

Stephanolepis hispidus (Linnaeus, 1758) - LIUFS 190 (1: 81,2 mm), LIUFS 266 (1: 45,7 mm)

Ostraciidae (FISCHER 1978; CERVIGÓN et al. 1992)

Acanthostracion quadricornis (Linnaeus, 1758) - LIUFS 500 (1:26,9 mm)

Lactophrys trigonus (Linnaeus, 1758) - LIUFS 453 (3: 13,8-22,5 mm)

Tetraodontidae (FISCHER 1978; LEMA et al. 1979; CERVIGÓN et al. 1992)

Lagocephalus laevigatus (Linnaeus, 1766) - LIUFS 204 (1: 94,8 mm)

Sphoeroides sp. - LIUFS 071 - (1: 72,4 mm), LIUFS 198 (3: 46,6-68,1 mm),

LIUFS 239 (1: 99,8 mm), LIUFS 254 (2: 59,2-68,8 mm)

Sphoeroides greeleyi Gilbert, 1900 - LIUFS 105 (1: 82,9 mm), LIUFS 174

(2: 79,6-95,9 mm), LIUFS 194 (1: 47,6 mm), LIUFS 294 (1: 44,8 mm),

LIUFS 320 (4: 30,9-37,3 mm)

Sphoeroides spengleri (Bloch, 1785) - LIUFS 062 (3: 43,2-62,9 mm)

Sphoeroides testudineus (Linnaeus, 1758) - LIUFS 103 (1: 85,6 mm),

LIUFS 173 (2: 88,1-89,2 mm), LIUFS 214 (1: 133,1 mm), LIUFS 228

(1: $76,9 \mathrm{~mm})$

Diodontidae (FISCHER 1978; CERVIGÓN et al. 1992)

Chilomycterus antillarum Jordan \& Rutter, 1897 - LIUFS 082 (2: 22,1-41,4 $\mathrm{mm})$, LIUFS 263 (1: 42,7 mm)

\section{DISCUSSÃO}

As florestas de manguezais cobrem, nos países da América Latina, 3.017.725 hectares dos quais 1.376.255 hectares situam-se no Brasil desde o Amapá (Oiapoque) até Santa Catarina (Praia do Sonho) e apresentam uma fauna bastante característica, em parte, exclusiva deste ecossistema. Outras espécies passam aí parte do seu ciclo de vida como, por exemplo, os peixes, Centropomus undecimalis, Micropogonias furnieri (Desmarest, 1823), Elops saurus Linnaeus, 1766, Megalops atlanticus Valenciennes, 1846 e Lutjanus griseus, apreciados como alimento ou na pesca esportiva (MOYLE \& CECH 1996; RAMOS E Silva 1996).

Os manguezais fornecem refúgio natural para indivíduos jovens contra predadores devido à proteção fornecida pela estrutura radicular de suas árvores; de modo geral, a maior parte dos peixes e crustáceos capturados nas áreas litorâneas tropicais desfruta desta proteção durante sua fase jovem e na época da postura e, assim, dependem intimamente da integridade deste ecossistema (LACERDA 1984). Deste modo, fica evidente a importância dos manguezais não só como verdadeiros "berçários" mas também como exportadores de matéria orgânica e geradores de recursos naturais primários para populações humanas locais (LACERDA 1984; SASEKUMAR et al. 1992; MOYLE \& CECH 1996). 
A maior parte do material coletado está constituído por indivíduos de pequeno porte, corroborando LACERDA (1984), MOYLE \& CECH (1996) e RAMOS \& Silva (1996) no que se refere à presença de formas jovens, de pequeno porte, em manguezais embora evidenciando também a limitação do apetrecho de pesca utilizado e o horário em que as coletas foram realizadas (somente durante a baixa-mar).

No que se refere às espécies de peixes coletadas como indivíduos jovens no manguezal de Cacha Pregos, entre suas formas adultas algumas são habitantes de recifes de corais como C. striatus, P. paru e Sparisoma sp. (R $\mathrm{R}^{\mathrm{ANDALL}} 1983$; MENEZES \& Figueiredo 1985). Outras vivem em áreas de substrato rochoso com F. tabacaria, S. plumieri e membros de Serranidae e Lutjanidae (FIGUEIREDo \& Menezes 1980; Menezes \& Figueiredo 1980; Allen 1985; Heemstra \& RaNDALL 1993). Em áreas de substrato arenoso habitam P. maculatus e $M$. curema (MENEZES \& FIGUEIREDO 1985) e em áreas de substrato lamoso e regiões estuarinas vivem $X$. brasiliensis, Centropomus spp. e membros de Gerreidae (FIGUEIREDO \& Menezes 1978, 1980; Menezes \& Figueiredo 1980). Membros de Clupeidae, S. barracuda e S. brasiliensis apresentam hábitos pelágicos (FIGUEIREDO \& MENEZES 1978; Collette \& Nauen 1983; Menezes \& Figueiredo 1985). Evidencia-se, assim, a ampla diversidade de ambientes ocupados posteriormente pela ictiofauna que habita e depende diretamente do manguezal de Cacha Pregos nas fases iniciais do seu ciclo de vida.

Além disso, dentre as formas jovens de espécies coletadas no manguezal de Cacha Pregos encontram-se várias de valor comercial como alimento quando adultas (por exemplo, Centropomus spp., E. itajara, Lutjanus spp. e S. brasiliensis) (Figueiredo \& Menezes 1980; Menezes \& Figueiredo 1980; Collette \& NAUEN 1983; HeEMSTRA \& RANDAll 1993). Epinephelus itajara, em particular, é considerada por RosA \& MENEZES (1996) como vulnerável dentre as espécies de peixes ameaçadas de extinção no Brasil.

AGRAdECIMENTOS. Ao Prof. Édson M. dos Santos (Departamento de Letras e Artes, Universidade Estadual de Feira de Santana) pela correção da versão do resumo para a língua inglesa. A todos que colaboraram nas coletas realizadas e em especial a Nilson A. Coelho pelo auxílio proporcionado.

\section{REFERÊNCIAS BIBLIOGRÁFICAS}

ALLEN, G.R. 1985. FAO species catalogue. Snappers of the world. An annotated and illustrated catalogue of lutjanid species known to date. FAO Fish. Synop. 6 (125): 1-208.

BAHIA PeSCA. 1994. Perfil do setor pesqueiro (litoral do Estado da Bahia). Salvador, Governo do Estado da Bahia, Secretaria da Agricultura, 75p.

BohlKe, J.E. \& C.C.G. ChAPlin. 1993. Fishes of the Bahamas and adjacent tropical waters. Austin, University of Texas Press, $2^{\text {nd }}$ ed., $771 \mathrm{p}$.

Carvalho, J.P.; L.R. Tommasi \& M.D. Novelli. 1968. Lista dos linguados do Brasil. Contr. Inst. Oceanogr. Univ. São Paulo (14): 1-26. 
CERVigón M., F. 1992. Fichas FAO de identificación de especies para los fines de la pesca. Guía de campo de las especies comerciales marinas y de aguas salobres de la costa septentrional de Sur America. Roma, Organización de las Naciones Unidas para la Agricultura y Alimentación, 595p.

Collette, B.B. \& C.E. NAuen. 1983. FAO species catalogue. Scombrids of the world. An annotated and illustated catalogue of tunas, mackerels, bonitos and related species known to date. FAO Fish. Synop. 2 (125): 1-137.

Figueiredo, J.L. 1977. Manual de peixes marinhos do sudeste do Brasil. I.

Introdução. Cações, raias e quimeras. São Paulo, Museu de Zoologia da Universidade de São Paulo, 104p.

Figueiredo, J.L. \& N.A. Menezes. Manual de peixes marinhos do sudeste do

Brasil. II. Teleostei (1). São Paulo, Museu de Zoologia da Universidade de São Paulo, 110p.

1980. Manual de peixes marinhos do sudeste do Brasil. III. Teleostei

(2). São Paulo, Museu de Zoologia da Universidade de São Paulo, 90p.

FISCHER, W. 1978. FAO species identification sheets for fishery purposes.

Western Central Atlantic (fishing area 31). Rome, Food and Agriculture Organization of the United Nations, 7 volumes não paginados.

HeEmSTRA, P.C. \& J.E. RandALL. 1993. FAO species catalogue. Vol. 16. Groupers of the world (family Serranidae, Subfamily Epinephelinae). An annotated and illustrated catalogue of the grouper, rockcod, hind, coral grouper and lyretail species known to date. FAO Fish. Synop. 16 (125): 1-382.

IHERING, R.V. 1931. Cyprinodontes brasileiros (peixes "guarús"). Systematica e informações biológicas. I. Arch. Inst. Biol. 4: 243-284.

LACERDA, L.D. 1984. Florestas de beira-mar. Ciência Hoje 3 (13): 62-70.

Lema, T.; C.A.S. Lucena; S. Saenger \& M.F.T. Oliveira. 1979. Primeiro levantamento dos Tetraodontiformes do extremo sul do Brasil, Uruguai e Argentina (Teleostei: Acanthopterygii). Comum. Mus. Ci. PUCRGS, Porto Alegre, (20): 1-84.

LOPES, P.R.D. \& R.A. MIRANDA. 1995. Notas sobre a alimentação de Ogcocephalus vespertilio (Linnaeus, 1758) (Teleostei, Ogcocephalidae) na localidade de Cacha Pregos (Ilha de Itaparica), Estado da Bahia. Acta Biol. Leopold. 17 (1): 87-94.

MENEZES, N.A. \& J.L. FigueIREDo. 1980. Manual de peixes marinhos do sudeste do Brasil. IV. Teleostei (3). São Paulo, Museu de Zoologia da Universidade de São Paulo, 96p.

1985. Manual de peixes marinhos do sudeste do Brasil. V. Teleostei

(4). São Paulo, Museu de Zoologia da Universidade de São Paulo, 105p.

MOYLE, P.B. \& J.J. CECH JR. 1996. Fishes. An introduction to Ichthyology. New Jersey, Prentice-Hall, $3^{\text {rd }}$ ed., 590p.

Nelson, J.S. 1994. Fishes of the world. New York, John Wiley \& Sons, 600p.

PIETSCH, T.W. 1984. The genera of frogfishes. Copeia 1984 (1): 27-44.

Ramos E Silva, C.A. 1996. Manguezal: ecossistema egoísta ou benevolente?

Ciência Hoje 20 (120): 6-11. 
RANDALL, J.E. 1983. Caribbean reef fishes. Hong Kong, T.F.H. Publications, $2^{\text {nd }}$ ed., 350p.

RIVAS, L.R. 1986. Systematic review of the perciform fishes of the genus Centropomus. Copeia 1986 (3): 579-611.

RosA, R.S. \& N.A. MENEZES. 1996. Relação preliminar das espécies de peixes (Pisces, Elasmobranchii, Actinopterygii) ameaçadas no Brasil. Revta bras. Zool. 13 (3): 647-667.

SANTOS, J.J. 1973. Estudo preliminar, principalmente do plâncton, das águas da Baía de Todos os Santos. Bol. Zool. Biol. Mar., N. S. (30): 419-447.

Sasekumar, A.; A.V.C. ChOnG; M.U. LeH \& R. D'CRUS. 1992. Mangroves as a habitat for fish and prawns, p. 195-207. In: V. JACCARINI \& E. MARTENS (Eds). The ecology of mangrove and related ecosystems. Kluwer Academic Publishers. Hydrobiologia 247: 195-207.

SoAres, L.H. 1978. Estudo dos Bothidae, Cynoglossidae e Soleidae, capturados nos bancos de camarão, no Estado do Rio Grande do Norte - Brasil. Bol. Dep. Ocean. Limnol. Centr. Bioc. Univ. Fed. Rio Grande Norte 6: 16-27.

Topp, R.W. \& F.W. HofF JR. 1972. Flatfishes (Pleuronectiformes). Mem. Hourglass Cruises 4 (2): 1-135.

WhiteHEAD, P.J.P. 1985. FAO species catalogue. Clupeoid fishes of the world (Suborder Clupeoidei). An annotated and illustrated catalogue of the herrings, sardines, pilchards, sprats, anchovies and wolf-herrings. Part 1 - Chirocentridae, Clupeidae and Pristigasteridae. FAO Fish. Synop. 7 (125): 1-303.

WhiteHEAD, P.J.P.; G.J. NELSON \& T. WongratANA. 1988. FAO species catalogue. Clupeoid fishes of the world (Suborder Clupeoidei). An annotated and illustrated catalogue of the herrings, sardines, pilchards, sprats, anchovies and wolf-herrings. Part 2. Engraulididae. FAO Fish. Synop. 7 (125): 305-579.

Recebido em 27.III.1997; aceito em 11.V.1998. 